\title{
Time-Domain Geometric Eddy-Current $A$ Formulation for Hexahedral Grids
}

\author{
Lorenzo Codecasa $^{1}$, Patrick Dular ${ }^{2}$, Ruben Specogna ${ }^{3}$, and Francesco Trevisan ${ }^{3}$ \\ ${ }^{1}$ Dipartimento di Elettronica ed Informazione, Politecnico di Milano, Milano 20133, Italy \\ ${ }^{2}$ Department of Electrical Engineering and Computer Science, Université de Liège, Liège 4000, Belgium \\ ${ }^{3}$ Dipartimento di Ingegneria Elettrica, Gestionale e Meccanica (DIEGM), Università di Udine, Udine 33100, Italy
}

\begin{abstract}
The aim of this paper is to present a 3-D time-domain eddy-current $\boldsymbol{A}$ formulation based on the discrete geometric approach (DGA) over unstructured and nonorthogonal hexahedral dual grids. The resulting differential algebraic system of equation, solved by means of a singly-diagonally implicit Runge-Kutta (SDIRK) variable step-size solver, leads to very accurate results at reduced computational costs, as shown by numerical analysis.
\end{abstract}

Index Terms-Cell method (CM), discrete geometric approach (DGA), eddy currents, finite integration technique (FIT), time domain.

\section{INTRODUCTION}

$\mathbf{T}$ HE so-called discrete geometric approach (DGA) [1], common to the finite integration technique (FIT) [2], [3] or the cell method (CM) [4], [5], allows to solve directly Maxwell's equations in an alternative way with respect to the classical Galerkin method in finite elements.

In [6], Codecasa et al. have proposed a novel method for discretizing the constitutive relations of the DGA for generic hexaedral dual grids. As a major theoretical result, such method guarantees the consistency and the stability of the discretized equations, in the sense of Lax-Richtmyer equivalence theorem [7].

In this paper, such novel method for discretizing constitutive relations is applied to eddy-current problems in the time domain. In this way, an $A$ formulation for eddy-current problems in the time domain is derived by the DGA, for unstructered and nonorthogonal dual grids, in which the primal grid is hexaedral. As far as the authors know, this is a major achievement with respect to previous results reported in literature [8], [9], in which eddy-current problems were discretized by DGA only over structured hexahedral grids. As shown by the proposed numerical analysis, the novel constitutive relations can lead to very accurate results at reduced computational costs, since they avoid the geometric discretization inaccuracy deriving from the use of orthogonal hexahedral grids [8], such as staircase effects, or from the use of structured hexahedral grids for modeling complex geometries [9].

\section{DGA FORMULATION}

The 3-D domain of interest $D$ of the eddy-current problem is covered by a mesh of generic hexahedra. The corresponding cell complex [4] is denoted as $\mathcal{K}$. Three subdomains of $D$ are identified: the passive conductive region $D_{c}$, the nonconductive

Manuscript received December 18, 2009; revised February 23, 2010; accepted February 26, 2010. Current version published July 21, 2010. Corresponding author: R. Specogna (e-mail: ruben.specogna@uniud.it).

Color versions of one or more of the figures in this paper are available online at http://ieeexplore.ieee.org.

Digital Object Identifier 10.1109/TMAG.2010.2044766 region (air region) $D_{a}$, and the source region $D_{s}$. From $\mathcal{K}$, a dual complex $\tilde{\mathcal{K}}$ is also introduced [4], based on the barycentric subdivision of the boundary of each hexahedron [10]. The incidence matrix between faces and edges is denoted by $\mathbf{C}$ and the incidence matrix between hexahedra and faces is denoted by $\mathbf{D}$. The incidence matrix between faces and edges of the dual complex is $\tilde{\mathbf{C}}=\mathbf{C}^{T}[4]$.

Next, the following integrals of the field quantities with respect to the oriented geometric elements of the mesh are introduced, yielding the degrees of freedom (DoF) arrays:

- $\boldsymbol{\Phi}$ is the array of magnetic fluxes associated with faces $f \in$ $D$;

- $\mathbf{F}$ is the array of magnetomotive forces (m.m.f.s) associated with dual edges $\tilde{e} \in D$;

- A is the array of circulations of the magnetic vector potential $A$ along primal edges $e \in D$;

- $\mathbf{I}$ is the array of currents associated with dual faces $\tilde{f}$ in $D_{c}$;

- array $\mathbf{I}_{s}$ of impressed currents associated with dual faces $\tilde{f}$ is introduced in $D_{s}$;

- finally $\mathbf{U}$ is the array of electromotive forces (e.m.f.s) on primal edges $e \in D_{c}$.

Maxwell's laws are written exactly as topological balance equations between DoFs arrays, as

$$
\begin{aligned}
(\tilde{\mathbf{C}} \mathbf{F})_{e} & =0, \quad e \in D_{a} \\
(\tilde{\mathbf{C}} \mathbf{F})_{e} & =\left(\mathbf{I}_{s}\right)_{e}, \quad e \in D_{s} \\
(\tilde{\mathbf{C}} \mathbf{F})_{e} & =(\mathbf{I})_{e}, \quad e \in D_{c} \\
(\boldsymbol{\Phi})_{f} & =(\mathbf{C A})_{f}, \quad f \in D
\end{aligned}
$$

where (1) is the Ampère law, and (2) involves the array $\mathbf{A}$ in such a way that Gauss' law $\mathbf{D} \boldsymbol{\Phi}=0$ is satisfied identically (since $\mathbf{D C}=0)$. Discrete Faraday's law

$$
(\mathbf{C U})_{f}=-\frac{\mathrm{d}}{\mathrm{d} t}(\boldsymbol{\Phi})_{f}, \quad e \in D_{c}
$$

is formulated, in terms of $\mathbf{A}$, as follows:

$$
(\mathbf{U})_{e}=\left(-\frac{\mathrm{d} \mathbf{A}}{\mathrm{d} t}\right)_{e}, \quad e \in D_{c} .
$$

The discrete counterpart of the constitutive laws is approximate and is written as

$$
\begin{aligned}
\mathbf{F} & =\boldsymbol{\nu} \boldsymbol{\Phi} \\
\mathbf{I} & =\boldsymbol{\sigma} \mathbf{U}
\end{aligned}
$$




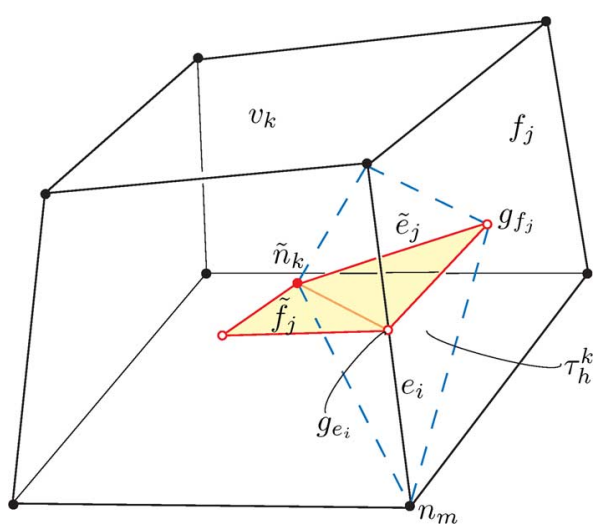

Fig. 1. Hexahedron $v_{k}$ is shown, together with a primal node $n_{m}$, a primal edge $e_{i}$, and a primal face $f_{j}$, and a dual node $\tilde{n}_{k}$, a dual edge $\tilde{e}_{i}$, and a dual face $\tilde{f}_{i}$. The barycenters $g_{e_{i}}$ of edge $e_{i}$ and $g_{f_{j}}$ of face $f_{j}$ are also shown. Also the tetrahedron $\tau_{h}^{k}$ is evidenced.

where $\boldsymbol{\nu}$ and $\boldsymbol{\sigma}$ are square symmetric positive-definite matrices ensuring consistency of equations, in the sense of Lax-Richtmyer equivalence theorem [7], and are constructed for an hexahedral primal grid in a novel way as outlined in the next section.

A symmetric algebraic system of equations, having $\mathbf{A}(t)$ as unknown, can be obtained by combining (2), (4), (5), and (6) into (1)

$$
\begin{aligned}
& \left(\mathbf{C}^{T} \boldsymbol{\nu} \mathbf{C A}(t)\right)_{e}=0, \quad e \in D_{a} \\
& \left(\mathbf{C}^{T} \boldsymbol{\nu} \mathbf{C A}(t)\right)_{e}=\mathbf{I}_{s}(t)_{e}, \quad e \in D_{s} \\
\left(\mathbf{C}^{T} \boldsymbol{\nu} \mathbf{C A}(t)\right)_{e}+ & \left(\boldsymbol{\sigma} \frac{\mathrm{d}}{\mathrm{d} t} \mathbf{A}_{c}(t)\right)_{e}=0, \quad e \in D_{c}
\end{aligned}
$$

where $\mathbf{A}_{c}$ contains the entries of the array $\mathbf{A}$ relative to the edges of $D_{c}$. The source current vector $\mathbf{I}_{s}(t)$ can be expressed, for example, as $\mathbf{I}_{s}(t)=\mathbf{I}_{s} \cdot s(t),{ }^{1}$ where $\mathbf{I}_{s}$ can be computed as described in [13] for a unit current and $s(t)$ is a function of time that describes the time evolution of the source current.

\section{A. Integral Sources}

When modeling stranded coils, it is useful to introduce integral sources, which do not require the coils to be meshed. With this aim, we express the array $\mathbf{A}$ as $\mathbf{A}=\mathbf{A}_{0}+\mathbf{A}_{r}$, where $\mathbf{A}_{0}$ contains the contribution produced by the source currents in $D_{s}$ and $\mathbf{A}_{r}$ due to the eddy currents in $D_{c}$.

Equation (7) can then be rewritten as

$$
\begin{gathered}
\left(\mathbf{C}^{T} \boldsymbol{\nu} \mathbf{C A}_{r}(t)\right)_{e}=0, \quad e \in D_{a} \cup D_{s} \\
\left(\mathbf{C}^{T} \boldsymbol{\nu} \mathbf{C A}_{r}(t)\right)_{e}+\left(\boldsymbol{\sigma} \frac{\mathrm{d}}{\mathrm{d} t} \mathbf{A}_{r c}(t)\right)_{e}=-(\mathbf{w}(t))_{e}, \quad e \in D_{c}
\end{gathered}
$$

where $(\mathbf{w}(t))_{e}=\left(\boldsymbol{\sigma} \mathbf{A}_{0 c}(\mathrm{~d} s(t) / \mathrm{d} t)\right)_{e}$. Each entry $\left(\mathbf{A}_{0 c}\right)_{e}$ of the array $\mathbf{A}_{0 c}$ can be precomputed as $\left(\mathbf{A}_{0 c}\right)_{e}=\int_{e} \mathrm{~A}_{0} \cdot d \mathrm{l}$, where $e$ is a primal edge in $D_{c}$ and $\mathrm{A}_{0}$ is the magnetic vector potential due to a unit source current in $D_{s}$.

\section{Discrete CONSTITUTIVE RELATIONS}

Each hexahedron $v_{k}$ of the primal grid is partitioned in 24 tetrahedra $\tau_{h}^{k}$, with $h=1, \ldots, 24$ (Fig. 1). Each tetrahedron

\footnotetext{
${ }^{1}$ In general, this technique can be easily extended to more than one coil.
}

$\tau_{h}^{k}$ has as vertices the dual node $\tilde{n}_{k}$ corresponding to $v_{k}$, the barycenter $\mathrm{g}_{f_{j}}$ of a primal face $f_{j}$ on the boundary of $v_{k}$, and the two primal nodes bounding a primal edge $e_{i}$ on the boundary of $f_{j}$. The label of the $i$ th primal edge is a function of the label of the $h$ th tetrahedron $\tau_{h}^{k}$ as $i=l^{k}(h)$. Similarly the label of the $j$ th primal face of $v_{k}$ is a function of the label of the $h$ th tetrahedron $\tau_{h}^{k}$ as $j=f^{k}(h)$.

In the generic tetrahedron $\tau_{h}^{k}$, we introduce a pair of triangles $s_{h}^{k}$ and $S_{h}^{k}$. The triangle $s_{h}^{k}$ has as vertices the dual node $\tilde{n}_{k}$, the barycenter of face $f_{j}$, with $j=f^{k}(h)$, and the barycenter of edge $e_{i}$, with $i=f^{k}(h)$. It is oriented as the dual face $\tilde{f}_{i}$, with $i=l^{k}(h)$. The triangle $S_{h}^{k}$ has as vertices the pair of nodes bounding edge $e_{i}$, with $i=f^{k}(h)$ and the barycenter of face $f_{j}$, with $j=f^{k}(h)$. It is oriented as the primal face $f_{j}$, with $j=f^{k}(h)$.

The following quantities are introduced, denoted in roman type: $\mathrm{e}_{i}$ is the edge vector ${ }^{2}$ corresponding to $e_{i}, \mathrm{f}_{j}$ is the face vector ${ }^{3}$ corresponding to $f_{j}, \tilde{\mathrm{e}}_{j}^{k}$ is the edge vector of the portion of dual edge $\tilde{e}_{j}$ contained in volume $v_{k}$, and $\tilde{f}_{i}^{k}$ is the face vector of the portion of dual face $\tilde{f}_{i}$ contained in volume $v_{k}$. We will also introduce the area vectors $\mathrm{s}_{h}^{k}, \mathrm{~S}_{h}^{k}$ associated with $s_{h}^{k}, S_{h}^{k}$, respectively.

We can now define the following piecewise uniform vector function $\mathrm{v}_{i}^{e}(p)$ attached to the edge $e_{i}$, defined at each point $p \in \tau_{h}^{k}$, as:

$$
\mathrm{v}_{i}^{e}(p)=\frac{\mathrm{s}_{h}^{k}}{3\left|\tau_{h}^{k}\right|} \delta_{i l^{k}(h)}+\left(\frac{\tilde{\mathrm{f}}_{i}^{k}}{\left|v_{k}\right|}-\frac{\mathrm{s}_{h}^{k}}{3\left|\tau_{h}^{k}\right|} \mathrm{e}_{l^{k}(h)} \cdot \frac{\tilde{\mathrm{f}}_{i}^{k}}{\left|v_{k}\right|}\right)
$$

where $\left|v_{k}\right|$ is the volume of $v^{k}$ and $\delta$ is the Kronecker delta symbol. We can also define the following piecewise uniform vector function $\mathrm{v}_{j}^{f}(p)$ attached to the face $f_{j}$, defined at each point $p \in \tau_{h}^{k}$ as

$$
\mathrm{v}_{j}^{f}(p)=\frac{\tilde{\mathrm{e}}_{f^{k}(h)}^{k} \xi_{h}^{k}}{3\left|\tau_{h}^{k}\right|} \delta_{j f(h)}+\left(\frac{\tilde{\mathrm{e}}_{j}^{k}}{\left|v_{k}\right|}-\frac{\mathrm{s}_{h}^{k}}{3\left|\tau_{h}^{k}\right|} \mathrm{f}_{f^{k}(h)} \cdot \frac{\tilde{\mathrm{e}}_{j}^{k}}{\left|v_{k}\right|}\right)
$$

where $\xi_{h}^{k}=\left|\mathrm{S}_{h}^{k}\right| /\left|\mathrm{f}_{f^{k}(h)}\right|$.

A show in [6], these vector functions, constructed in a purely geometric way, possess the properties requested for constructing discrete constitutive relations for DGA by means of the energetic approach [11], [12].

Thus, the following numbers:

$$
\begin{gathered}
\boldsymbol{\sigma}_{i j}=\int_{D_{c}} \mathrm{v}_{i}^{e} \cdot \sigma \mathrm{v}_{j}^{e} d v \\
\boldsymbol{\nu}_{i j}=\int_{D} \mathrm{v}_{i}^{f} \cdot \nu \mathrm{v}_{j}^{f} d v
\end{gathered}
$$

are the $i, j$ entries of symmetric positive-definite constitutive matrices $\sigma, \boldsymbol{\nu}$, respectively, ensuring consistency and stability of discrete equations.

${ }^{2}$ Edge vector $\mathrm{e}_{i}$ is directed as the edge $e_{i}$, its amplitude is the length of $e_{i}$, and it points as the inner orientation of the edge.

${ }^{3}$ Area vector $\mathbf{f}_{j}$ is normal to face $f_{j}$, its amplitude is the area of $f_{j}$, and it points in a way congruent with the screw rule with respect to the inner orientation of the face. 
Since the edge and face vector functions are piecewise uniform in $v_{k}$ (i.e., uniform in each $\tau_{h}^{k}$ subregion), the volume integrals in (11) and (12) can be efficiently computed

$$
\begin{aligned}
\boldsymbol{\sigma}_{i j} & =\sum_{h k} \mathrm{v}_{i}^{e}\left(p_{h}^{k}\right) \cdot \sigma \mathrm{v}_{j}^{e}\left(p_{h}^{k}\right)\left|\tau_{h}^{k}\right| \\
\nu_{i j} & =\sum_{h k} \mathrm{v}_{i}^{f}\left(p_{h}^{k}\right) \cdot \nu \mathrm{v}_{j}^{f}\left(p_{h}^{k}\right)\left|\tau_{h}^{k}\right|
\end{aligned}
$$

where $\left|\tau_{h}^{k}\right|$ is the volume of $\tau_{h}^{k}$ and $p_{h}^{k}$ is any point in $\tau_{h}^{k}$.

\section{Time InTEGRATION Method FOR DAE PROBLEM}

Systems (7) and (8) can be recast into the general form

$$
\mathbf{B y}^{\prime}=\mathbf{a}(\mathbf{y}, t) \mathbf{y}+\mathbf{f}(t)
$$

where array $\mathbf{y}(t)$ denotes one of the unknown arrays $\mathbf{A}(t)$ or $\mathbf{A}_{r}(t)$, and $\mathbf{B}$ and $\mathbf{a}$ are square matrices of dimension $L, L$ being the number of primal edges in $D$. Their definition can be easily evinced from (7) and (8). In our eddy-current problem, matrix $\mathbf{a}(\mathbf{y}, t)$ is time invariant and independent of $\mathbf{y}$, the magnetic medium being linear. Matrix $\mathbf{B}$ is time invariant and singular. In this way, (14) is not an ordinary differential equation (ODE) but rather a system of differential algebraic equations (DAEs) of $C^{2}$ class. To solve (7) or (8), we rely on an inhouse developed singly-diagonally implicit Runge-Kutta (SDIRK) DAE solver with a variable step size. In the following sections, we will summarize the implemented algorithm based on the fundamental papers [14]-[17].

\section{A. SDIRK DAE Solver}

We focus on a Runge-Kutta (R-K) method with $s=4$ stages. In Fig. 2, we introduce an $s \times s$ matrix $\mathbf{A}$, and $s \times 1$ arrays $\mathbf{b}$, $\mathbf{c}=\mathbf{A e}_{s}$, where $\mathbf{e}_{s}$ is an array of ones $s \times 1$. The components of $\mathbf{b}$ and $\mathbf{c}$ are referred to as weights and abscissae, respectively. Starting from $\left\{\mathbf{y}_{n}, t_{n}\right\}$, the $i$ th stage of an R-K method $(\mathbf{A}, \mathbf{b})$ is computed as

$$
\mathbf{Y}_{i}=\mathbf{y}_{n}+h \sum_{j=1}^{s} a_{i j} \mathbf{Y}_{j}^{\prime}, \quad i=1, \ldots, s
$$

where $\mathbf{Y}_{i}$ is the stage value, $\mathbf{Y}_{i}^{\prime}$ is the stage derivative, and $h$ is the current step. The new estimate $\mathbf{y}_{n+1}$ at $t_{n+1}=t_{n}+h$ is updated by

$$
\mathbf{y}_{n+1}=\mathbf{y}_{n}+h \sum_{i=1}^{s} b_{i} \mathbf{Y}_{i}^{\prime}
$$

To apply an R-K method, the following substitutions in (14) are made for the stage $i$ : $\mathbf{y}$ is replaced by $\mathbf{Y}_{i}$ given by (15), $\mathbf{y}^{\prime}$ by $\mathbf{Y}_{i}^{\prime}$, and $t$ by $t_{n}+h c_{i}$. Thus, we obtain the following stage equation:

$$
\mathbf{F}_{i}\left(\mathbf{Y}_{i}^{\prime}, \mathbf{y}_{n}+h \sum_{j=1}^{s} a_{i j} \mathbf{Y}_{j}^{\prime}\right)=0, \quad i=1, \ldots, s .
$$

Since in an SDIRK matrix $\mathbf{A}$ is lower triangular, by introducing $\mathbf{W}=\mathbf{A}^{-1}$, we can invert (15) yielding the stage derivative
$\mathbf{Y}_{i}^{\prime}$ in terms of the stage variables $\mathbf{Y}_{i}$ and the stage (17) can be rewritten as

$$
\mathbf{B}\left[\sum_{j=1}^{i} w_{i j}\left(\mathbf{Y}_{j}-\mathbf{y}_{n}\right)\right]-h \mathbf{a} \mathbf{Y}_{i}-h \mathbf{f}\left(t_{n}+h c_{i}\right)=0 .
$$

In the general nonlinear case, this stage equation is solved by means of Newton's method with stage Jacobian $\mathbf{J}_{i}=w_{i i} \mathbf{B}-$ $h \mathbf{a}$. One can freely update a Jacobian to improve performance or keep the same Jacobian for several stages if it gives acceptable convergence. However, in our linear case, a linear system can be conveniently used instead.

The solution is now updated as

$$
\mathbf{y}_{n+1}=\left(1-\mathbf{d}^{T} \mathbf{e}\right) \mathbf{y}_{n}+\sum_{i=1}^{s} d_{i} \mathbf{Y}_{i}
$$

where $\mathbf{d}^{T}=\mathbf{b}^{T} \mathbf{w}$ and $\mathbf{e}$ is a vector of ones $L \times 1$.

\section{B. Error Estimation}

An embedded pair $(\mathbf{A}, \mathbf{b})-(\mathbf{A}, \hat{\mathbf{b}})$ of $\mathrm{R}-\mathrm{K}$ methods is typically used to estimate the error in $\mathbf{y}$. Such a pair uses the same matrix $\mathbf{A}$ but different advancing vectors $\mathbf{b}$ and $\hat{\mathbf{b}}$ and related local orders ${ }^{4} k_{L}$ and $\hat{k}_{L}$. In the implemented $\operatorname{SDIRK},(\mathbf{A}, \mathbf{b})$ is accurate with $k_{L}=4$ and the auxiliary $\operatorname{SDIRK}(\mathbf{A}, \hat{\mathbf{b}})$ has $\hat{k}_{L}=3$. If $\mathbf{y}_{n+1}$ and $\hat{\mathbf{y}}_{n+1}$ are the estimates from these two methods, $k_{L}<\hat{k}_{L}$ holds, and the difference between them is typically assumed to be the local error for the $(\mathbf{A}, \mathbf{b})$ method

$$
\mathbf{e}_{n+1}=\mathbf{y}_{n+1}-\hat{\mathbf{y}}_{n+1} \text {. }
$$

Letting $\hat{\mathbf{d}}^{T}=\hat{\mathbf{b}}^{T} \mathbf{W}$, the local error for an embedded SDIRK pair is computed as

$$
\mathbf{e}_{n+1}=\left(\hat{\mathbf{d}}^{T}-\mathbf{d}^{T}\right) \mathbf{e y}_{n}+\sum_{i=1}^{s}\left(d_{i}-\hat{d}_{i}\right) \mathbf{Y}_{i} .
$$

\section{NUMERICAL EXPERIMENT}

A fully 3-D geometry consisting of a circular coil placed above a conducting plate with conductivity $\sigma_{c}=4 \cdot 10^{7} \mathrm{~S} / \mathrm{m}$ is considered as a benchmark problem. Such a simple geometry is chosen to be able to compare the results with an accurate reference solution obtained by using a 2-D axisymmetric independent code (ANSYS). The geometry is shown in Fig. 3. The considered primal grid is represented in Fig. 4, where domains $D_{c}, D_{s}$, and $D_{a}$ are shown. The source current is enforced by a stranded circular coil in $D_{s}$ with a time dependence $s(t)=400 \cdot\left(1-e^{-t / \tau}\right)$, where $\tau=1 \mathrm{~ms}$.

To compare the results obtained with the DGA implemented in the geometric approach to Maxwell's equations (GAME) code,, 5 the ANSYS finite-elements software is used to compute reference solutions. Since the problem is axisymmetric, a reference solution has been computed with ANSYS using a 2-D quadrilateral mesh consisting of about 50000 elements of second order (time step of $0.01 \mathrm{~ms}$ ).

Then, a fully 3-D simulation is computed with the GAME code using the primal grid consisting of 19136 hexahedra

${ }^{4} \mathrm{~A}$ time integration method is of order $p$ if the local error depends asymptotically on the time step $h$ as $O\left(h^{p+1}\right)$.

${ }^{5}$ http://www.comphys.com 


\begin{tabular}{|c|c|c|c|c|}
\hline c $1 / 4$ & $1 / 4$ & 0 & 0 & 0 \\
\hline $11 / 28$ & $1 / 7$ & $1 / 4$ & 0 & 0 \\
\hline $1 / 3$ & $61 / 144$ & $-49 / 144$ & $1 / 4$ & 0 \\
\hline 1 & 0 & 0 & $3 / 4$ & $1 / 4$ \\
\hline$\overline{\mathbf{b}^{T}}$ & 0 & 0 & $3 / 4$ & $1 / 4$ \\
\hline$\hat{\mathbf{b}}^{T}$ & $-61 / 144$ & $49 / 600$ & $79 / 100$ & $23 / 100$ \\
\hline
\end{tabular}

Fig. 2. Butcher table showing the arrays $\mathbf{A}, \mathbf{b}, \hat{\mathbf{b}}$, and $\mathbf{c}$.

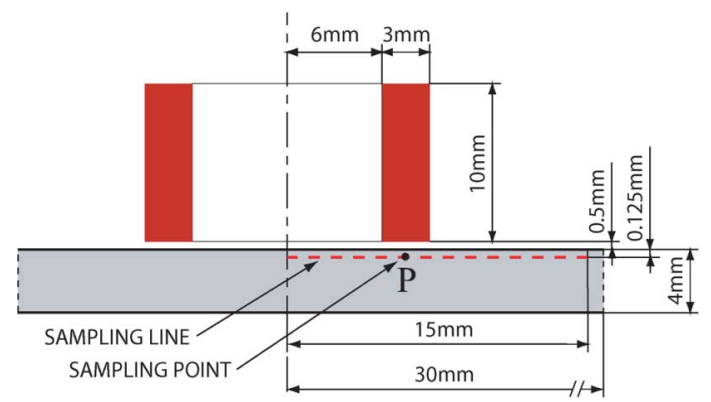

Fig. 3. Geometry of the benchmark problem, a circular coil above a conductive plate.

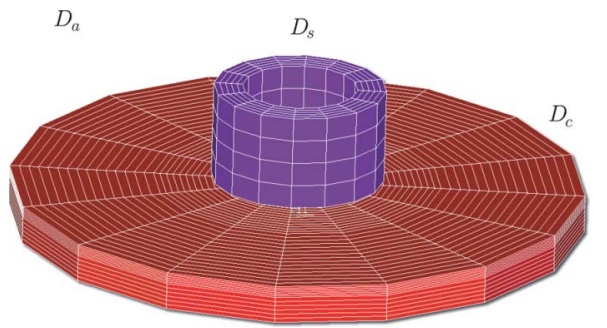

Fig. 4. Hexahedra of the considered primal grid belonging to $D_{c}$ and $D_{s}$ domains.

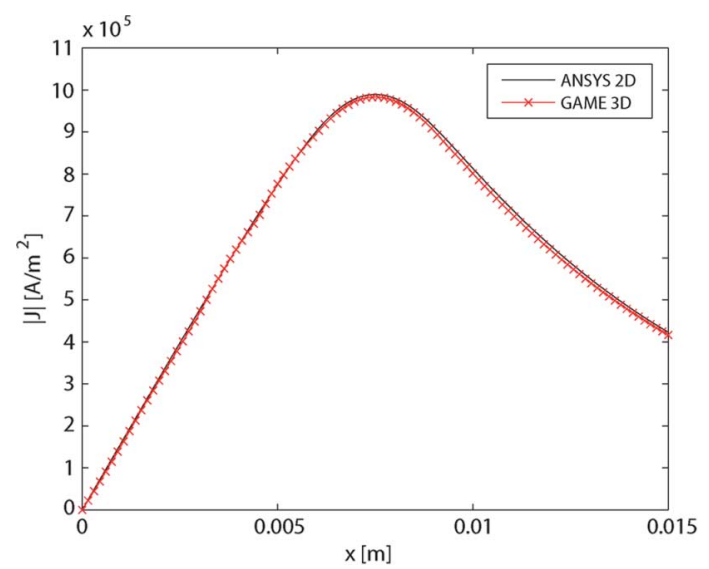

Fig. 5. Amplitude of the current densities along the sampling line shown in Fig. 3.

(59330 DoFs) shown in Fig. 4. The GAME takes about $4 \mathrm{~s}$ of CPU time for the assembling of the sparse matrices and $4 \mathrm{~s}$ for each time step. In the implemented DAE solver, the time step is variable.

The amplitude of the current density along a number of points evenly distributed along a sampling line (shown in Fig. 3) for $t=1 \mathrm{~ms}$ is represented in Fig. 5. In Fig. 6, the time behavior of the amplitude of the current density in the point $P$ (situated on the sampling line, at a distance of $7.5 \mathrm{~mm}$ from the axis) is shown. The results obtained by the GAME code are in a quite good agreement with those of ANSYS.

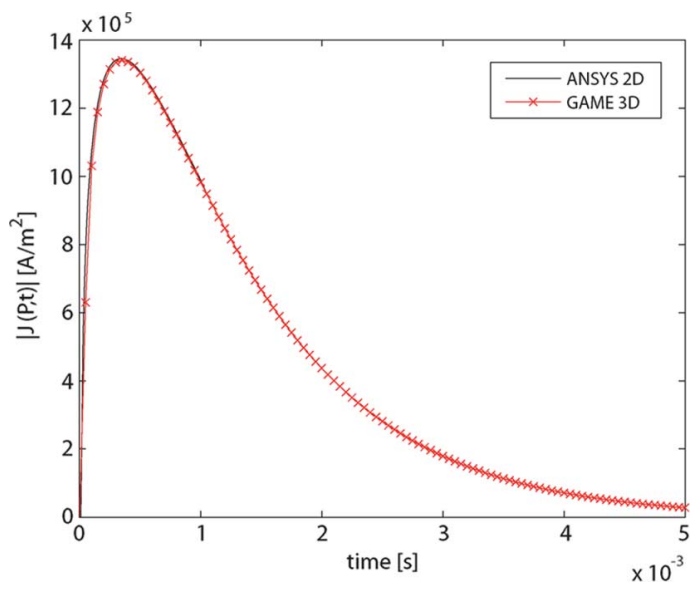

Fig. 6. Time evolution of the module of the current density in the reference point $P$ shown in Fig. 3 .

\section{CONCLUSION}

A 3-D geometric time-domain eddy-current $A$ formulation suitable with hexahedral meshes has been presented. The formulation has been successfully validated using a finite-elements commercial software.

\section{ACKNOWLEDGMENT}

The authors would like to thank M.Sc. Paolo Martin for a preliminary implementation of the DAE solver algorithm.

\section{REFERENCES}

[1] A. Bossavit, "How weak is the weak solution in finite elements methods?," IEEE Trans. Magn., vol. 34, no. 5, pp. 2429-2432, 1998.

[2] T. Weiland, "A discretization method for the solution of Maxwell's equations for six-component fields," Electron. Commun. (AEÜ), vol. 31, no. 3, p. 116, 1977.

[3] M. Clemens and T. Weiland, "Discrete electromagnetism with the finite integration technique," Progr. Electromagn. Res. (PIER) Monograph Ser., vol. 32, pp. 65-87, 2001.

[4] E. Tonti, "Algebraic topology and computational electromagnetism," in Proc. 4th Int. Workshop Electr. Magn. Fields, Marseille, France, May $12-15,1988$, pp. $284-294$.

[5] E. Tonti, "Finite formulation of the electromagnetic field," IEEE Trans. Mag., vol. 38, no. 2, pp. 333-336, 2002.

[6] L. Codecasa, R. Specogna, and F. Trevisan, "Constitutive relations for discrete geometric approach over hexahedral grids," IEEE Trans. Magn., accepted for publication.

[7] A. Bossavit and L. Kettunen, "Yee-like schemes on staggered cellular grids: A synthesis between FIT and FEM approaches," IEEE Trans. Magn., vol. 36, no. 4, pp. 861-867, 2000.

[8] M. Clemens, M. Wilke, and T. Weiland "3-D transient eddy-current simulations using $F I^{2} T D$ schemes with variable time-step selection," IEEE Trans. Magn., vol. 38, pp. 605-608, 2002.

[9] M. Clemens and T. Weiland, "Magnetic field simulation using conformal FIT formulations," IEEE Trans. Magn., vol. 38, pp. 389-392, 2002.

[10] L. Codecasa, R. Specogna, and F. Trevisan, "Discrete constitutive equations over hexahedral grids for eddy-current problems," Comput. Model. Eng. Sci., vol. 31, no. 3, pp. 129-144, 2008.

[11] L. Codecasa, R. Specogna, and F. Trevisan, "Symmetric positive-definite constitutive matrices for discrete eddy-current problems," IEEE Trans. Magn., vol. 43, pp. 510-515, 2007.

[12] L. Codecasa and F. Trevisan, "Piecewise uniform bases and energetic approach for discrete constitutive matrices in electromagnetic problems," Int. J. Numer. Meth. Eng., vol. 65, no. 4, pp. 548-565, 2006.

[13] R. Specogna and F. Trevisan, "Discrete constitutive equations in $A-\chi$ geometric eddy-currents formulation," IEEE Trans. Magn., vol. 41, no. 4, pp. 1259-1263, 2005.

[14] A. Nicolet and F. Delincé, "Implicit Runge-Kutta methods for transient magnetic field computation," IEEE Trans. Magn., vol. 32, no. 3, pp. 1405-1408, 1996.

[15] F. Cameron, R. Piché, and K. Forsman, "Variable step size time integration methods for transient eddy current problems," IEEE Trans. Magn., vol. 34, no. 5, pp. 3319-3322, 1998.

[16] F. Cameron, "Low-order Runge-Kutta methods for differential-algebraic equations," Ph.D. dissertation, Tampere Univ. Technol., Tampere, Finland, 1999.

[17] F. Cameron, M. Palmroth, and R. Piché, "Quasi stage order conditions for SDIRK methods," Appl. Numer. Math., vol. 4241, pp. 61-75, 2002. 